\title{
Vacuna contra el virus papiloma humano como tratamiento para la papilomatosis respiratoria recurrente
}

\author{
Human papilloma virus vaccination treatment \\ for recurrent respiratory papillomatosis
}

\author{
Andrés Rosenbaum F1, Antonia Lagos $\mathrm{V}^{2}$, Carla Napolitano V², Pedro Badía ${ }^{2}$.
}

\begin{abstract}
RESUMEN
Una de las manifestaciones clínicas del virus papiloma humano (VPH) es la papilomatosis respiratoria recurrente (PRR), que se caracteriza por la proliferación de lesiones epiteliales verrucosas recurrentes en la mucosa respiratoria, pudiendo progresar a obstrucción de vía aérea o presentar transformación maligna. El tratamiento de primera línea quirúrgico, pero dada su alta recurrencia ha tomado peso el tratamiento adyuvante, como la vacuna tetravalente contra VPH. Sin embargo, existe controversia respecto a su eficacia. El objetivo de esta revisión es analizar la efectividad de la vacuna contra VPH como tratamiento adyuvante de la PRR, para lo cual hicimos una revisión de la literatura sobre la efectividad de la vacuna tetravalente contra VPH para $P R R$, realizando una búsqueda en diversas fuentes: Pubmed, MEDLINE, EMBASE, Cochrane, Google Scholar y Epistemonikos. Se seleccionaron los estudios que responden a la pregunta y se analizaron los datos de los estudios primarios. Se encontraron cinco estudios primarios no aleatorizados, todos a favor de la vacuna como tratamiento. Concluimos con baja certeza de evidencia, que la vacuna es posiblemente efectiva para pacientes con PRR en disminuir el número de recurrencias, aumentar el intervalo entre cirugías, lograr remisión completa o parcial de la enfermedad y aumentar significativamente los títulos de anticuerpos anti-VPH.
\end{abstract}

Palabras clave: Virus papiloma humano, tratamiento, vacuna, papilomatosis respiratoria recurrente.

\begin{abstract}
A clinical manifestation of human papillomavirus (HPV) is recurrent respiratory papillomatosis (RRP), characterized by the proliferation of recurrent verrucous epithelial lesions in the respiratory mucosa, which may progress to airway obstruction or malignant transformation. First-line treatment is surgery, but given its high recurrence the use of adjuvant therapy, such as the quadrivalent vaccine, has gained importance. However, there is controversy regarding its effectiveness. To analyze the effectiveness of the HPV
\end{abstract}

Interno de Medicia, Escuela de Medicina, Pontificia Universidad Católica de Chile.

2 Médicos Departamento de Otorrinolaringología, Pontificia Universidad Católica de Chile.

Recibido el 25 de octubre, 2017. Aceptado el 10 de diciembre, 2017. 
vaccine as an adjuvant treatment for RRP a review of the literature on the effectiveness of HPV tetravalent vaccine for RRP was performed by searching databases such as Pubmed, MEDLINE, EMBASE, Cochrane, Google Scholar and Epistemonikos. We selected the studies that answered the question and analyzed the data from all of which supported the vaccine as treatment. None were randomized controlled trials. We conclude, with low certainty of evidence, that the vaccine is possibly effective for RRP in decreasing the number of recurrences, increasing the intersurgical interval, achieving complete or partial remission of disease and significantly increasing anti-HPV antibodies.

Key words: Human papillomavirus, treatment, vaccine, recurrent respiratory papillomatosis.

\section{INTRODUCCIÓN}

El virus del papiloma humano (VPH) es la infección de transmisión sexual más frecuente en el mundo. Se han identificado más de 200 serotipos del virus, 40 de ellos vinculados a infección en humanos con distintos grados de oncogenicidad, siendo de mayor riesgo los serotipos 16 y 18 , y de bajo riesgo 6 y $11^{2}$. Si bien puede tener un curso autolimitado y ser asintomático, puede evolucionar a cáncer orofaríngeo, cérvico-uterino o rectal, y además puede manifestarse como verrugas genitales 0 papilomatosis respiratoria recurrente (PRR). Esta última se caracteriza por la proliferación de lesiones epiteliales verrucosas recurrentes en la mucosa respiratoria; su sitio predilecto de presentación es la laringe, donde se manifiesta como disfonía, pero puede progresar a obstrucción de la vía aérea 0 incluso presentar transformación maligna hasta en el $2 \%$ de los casos ${ }^{3}$.

La PRR tiene una incidencia estimada en adultos de 1,8 en 100.000 habitantes y de 4,3 cada 100.000 niños, siendo el tumor infantil de laringe más frecuente ${ }^{4,5}$. Presenta un peak de incidencia bimodal con formas clínicas características para cada grupo etáreo: la forma recurrente juvenil en el grupo de menores de 5 años, transmitido de madre a hijo durante el parto, que se caracteriza por ser más agresiva; y la forma tardía en adultos de 20-30 años que resulta habitualmente del contagio orogenital ${ }^{6}$. Además, existe una tercera forma clínica que es la papilomatosis juvenil con persistencia hacia la adultez ${ }^{7}$. La infección es causada en 95\% por los serotipos 6 y $11^{8}$, y tiene predilección por las zonas de transición entre epitelio respiratorio ciliado y escamoso. El área más frecuentemente afectada es la laringe, específicamente las cuerdas vocales, correspondiendo al $97,5 \%$ de las PRR. Además puede comprometer zonas extralaríngeas como esófago, tráquea, bronquios, pulmones, orofaringe y cavidad nasal ${ }^{6}$. El tratamiento de primera línea es la resección quirúrgica de las lesiones con láser, instrumentos fríos, 0 microdebridador. El curso de la enfermedad es variable, desde remisión luego de la primera intervención, hasta múltiples recurrencias que requieren reintervención cada 1-3 meses ${ }^{4}$. Recientemente ha tomado peso el tratamiento adyuvante indicado para prevenir recurrencias en casos agresivos que se han definido como aquellos que requieren 100 más cirugías en el tiempo, 30 más cirugías en 1 año, 0 si se extiende a la subglotis o más allá. Se han descrito como terapias adyuvantes el uso de interferón alfa (proteína antineoplásica e inmunomoduladora), cidofovir (antiviral que inhibe la ADN polimerasa del virus, impidiendo su replicación y transcripción), bevacizumab (anticuerpo monoclonal que se une al factor de crecimiento endotelial vascular-VEGF) y la vacuna tetravalente contra VPH (Gardasil $\left.{ }^{\circledR}\right)$. EI objetivo de esta revisión de la literatura es analizar la efectividad de la vacuna contra el VPH como tratamiento adyuvante de la PRR.

\section{VIRUS PAPILOMA HUMANO}

El VPH es un virus pequeño sin envoltura, que contiene ADN circular de doble hebra, cubierto por una cápside constituida por las proteínas estructurales L1 y L2. La primera es el mayor componente de la cápside y contiene el determinante requerido para la adhesión inicial a la superficie celular. L1 es muy inmunogénica, ya que contiene epítopes conformacionales que inducen 
la producción de anticuerpos específicos contra el virus que previenen la infección. Se encuentra altamente conservada entre los distintos serotipos virales. L2 es una proteína menor de la cápside de ADN, y contribuye a la unión del virión al receptor celular y su posterior transporte al núcleo celular para su replicación. Por lo tanto, la infección por el virus se basa en la adhesión inicial de L1 a la membrana basal expuesta de células que han sido sometidas a trauma, y luego se internaliza el virón hacia el núcleo celular mediante la función de L2. Inicialmente ocurre la replicación del ADN viral en el núcleo de las células basales del epitelio y posteriormente en las capas más superficiales ${ }^{10}$.

\section{FARMACOLOGÍA DE LA VACUNA}

Gardasi ${ }^{\circledR}$ es una vacuna recombinante tetravalente contra los serotipos 6, 11, 16 y 18 del VPH, que ha mostrado prevenir la transmisión de la enfermedad en individuos que han recibido la vacuna antes de la exposición al virus ${ }^{11}$. Fue diseñada para aumentar la inmunogenicidad contra la proteína de cápside viral L1. Se administra de forma intramuscular en 3 dosis, de $0,5 \mathrm{ml}$ cada una, con el esquema 0-2-6 meses. Se ha demostrado que la vacuna en pacientes infectados aumenta los títulos de anticuerpos a niveles mayores que los que se producen posterior a la infección natural12,13.

\section{EFECTOS ADVERSOS Y CONTRAINDICACIONES}

Según la FDA, Gardasi ${ }^{\circledR}$ tiene un perfil de efectos adversos relativamente bajos, no significativos, que incluyen más frecuentemente complicaciones locales como dolor, edema, eritema, prurito y sangrado ${ }^{14}$. Menos frecuentes son las complicaciones sistémicas como compromiso del estado general, fiebre, mareos, diarrea, dolor abdominal, convulsiones y síncope, por lo que se recomienda observación del paciente por al menos 15 minutos posterior a su administración. Se ha descrito una tasa levemente mayor de eventos tromboembólicos comparado con otras vacunas $(0,2$ casos por 100.000) ${ }^{15}$. Las contraindicaciones incluyen a pacientes con reacción de hipersensibilidad a dosis previas de la vacuna, levadura, polisorbato
80 o sulfato de aluminio. Además, es relevante considerar que el tratamiento inmunosupresor incluyendo radiación, drogas citotóxicas y corticoides sistémicos podrían reducir la respuesta inmunogénica de la vacuna.

\section{USOS ACTUALES}

Generalmente está indicada en niñas y mujeres desde los 9 a los 26 años de edad, previo al inicio de su actividad sexual, para la prevención de condilomas acuminados y cáncer cervicouterino, vulvar, vaginal y anal, así como sus respectivas lesiones intraepiteliales de bajo grado. También se recomienda su administración en niños y hombres entre 9 y 26 años, previo al inicio de su actividad sexual, para la prevención de cáncer anal y condilomas acuminados. Como se mencionó anteriormente, se ha planteado su uso en el ámbito otorrinolaringológico como tratamiento adyuvante en la PRR. El presente texto pretende realizar una revisión de la literatura respecto a la evidencia disponible actualmente sobre su efectividad en este último ámbito.

\section{EVIDENCIA DE GARDASIL ${ }^{\circledR}$ PARA PRR METODOLOGÍA DE LA BÚSQUEDA DE EVIDENCIA}

Se realizó una búsqueda de revisiones sistemáticas y estudios primarios en diversas fuentes como Pubmed, MEDLINE, EMBASE, Cochrane, Google Scholar y Epistemonikos con la siguiente estrategia: "recurrent respiratory papillomatosis" OR "recurrent laryngeal papillomatosis" AND "vaccine" OR "gardasil". Se seleccionaron los estudios que responden a la pregunta y analizamos los datos de los estudios primarios.

\section{RESULTADOS}

Se encontraron cinco estudios primarios que responden a la pregunta sobre la efectividad de la vacuna tetravalente $\left(\right.$ Gardasi $^{\circledR}{ }^{\circledR}$ ) como tratamiento adyuvante en papilomatosis respiratoria recurrente, los cuales se muestran en la Tabla 1. No se encontraron revisiones sistemáticas al respecto. 
Tabla 1. Características de los estudios primarios

\begin{tabular}{|c|c|c|c|c|}
\hline Estudio & Población & Intervención & Outcome & Diseño \\
\hline Chirla $2014^{16}$ & $\begin{array}{l}n=31 . \text { PRR con cidofovir } \\
+ \text { cirugía }\end{array}$ & $\begin{array}{l}\text { Vacuna tetravalente } \\
\text { anti-VPH en } 13 \text { pacientes }\end{array}$ & Recurrencia a 1 año & $\begin{array}{l}\text { Prospectivo no } \\
\text { randomizado }\end{array}$ \\
\hline Young $2015^{17}$ & $\mathrm{n}=20 . \mathrm{PRR}+$ cirugía & Gardasil $^{\circledast}$ (3 dosis) & $\begin{array}{l}\text { Intervalo libre de cirugía, } \\
\text { número remisión } \\
\text { completa o parcial }\end{array}$ & $\begin{array}{l}\text { Retrospectivo } \\
\text { observacional }\end{array}$ \\
\hline Tijon $2016^{13}$ & $n=6 . P R R$ & Gardasil $^{\circledR}$ (3 dosis) & $\begin{array}{l}\text { Seroactividad } \\
\text { (cápside L1) }\end{array}$ & $\begin{array}{l}\text { Retrospectivo } \\
\text { observacional }\end{array}$ \\
\hline Makiyama $2016^{18}$ & $\begin{array}{l}n=6 . \text { Papilomatosis } \\
\text { Laringea Recurrente }\end{array}$ & $\begin{array}{l}\text { Gardasil }{ }^{\otimes} \text { (3 dosis) } \\
+ \text { cirugía }\end{array}$ & $\begin{array}{l}\text { Títulos de VPH antes } \\
\text { y después de } 7 \text { meses }\end{array}$ & Serie de casos \\
\hline Goon $2017^{19}$ & $\begin{array}{l}n=12 . \text { PRR con } \\
\text { serotipos } 6 \text { y } 11 .\end{array}$ & $\begin{array}{l}\text { Gardasil }{ }^{\circledast} \text { (3 dosis) } \\
\text { + cirugía. }\end{array}$ & $\begin{array}{l}\text { Tasa de incidencia de } \\
\text { PRR que requiere cirugía } \\
\text { antes y } 6 \text { meses después }\end{array}$ & $\begin{array}{l}\text { Prospectivo no } \\
\text { randomizado }\end{array}$ \\
\hline
\end{tabular}

Un estudio prospectivo no aleatorizado realizado en Rumania por Chirla y col ${ }^{16}$ en 2014, de 31 pacientes con PRR en su presentación juvenil (diagnosticado en menores de 17 años) y adulta (diagnosticado en mayores de 17 años), utilizó

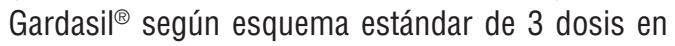
13 pacientes con papilomatosis severa (más de tres cirugías al año) que recurrió luego de tratamiento con resección quirúrgica y cidofovir, y se mantuvieron en seguimiento por 1 año. El estudio concluyó que el 85\% (IC 95\% [54,44-99,41]) de los pacientes intervenidos con la vacuna tetravalente no presentó evidencia de recurrencia a un 1 año de la intervención. Se observó recurrencia en solo dos pacientes (15\%, IC 95\% [0,59-14,56]), uno con presentación juvenil y otro adulto, ambos luego de la primera dosis de la vacuna, que fueron reintervenidos un mes posterior a la tercera dosis, sin recurrencias luego de 1 año de seguimiento.

Un estudio retrospectivo observacional realizado en Estados Unidos por Young y $\mathrm{col}^{17}$ en 2015, incluyó 20 pacientes con diagnóstico de PRR que recibieron cidofovir más tratamiento quirúrgico mediante escisión con láser 0 mircrodebridador, a quienes además se les administró Gardasil ${ }^{\circledR}$ en esquema estándar de 3 dosis. Los desenlaces medidos fueron el intervalo libre de cirugías y el número de pacientes con remisión completa 0 parcial, antes y después de recibir la vacuna. Se observó un aumento significativo de 3,1 meses en el intervalo libre de cirugías (IC 95\% [1,02 - 5,19]), mayor en pacientes de sexo masculino que femenino (4,2 meses vs 1,2 meses). Además, el $65 \%$ de los pacientes lograron remisión completa $(40 \%) 0$ parcial $(25 \%)$, concluyendo que la vacuna puede modular la severidad de la PRR e inducir remisión en algunos pacientes, con efecto mayor en sexo masculino.

Un estudio retrospectivo observacional llevado a cabo en Holanda por Tijon y col ${ }^{13}$ en 2016, incluyó 6 pacientes con PRR confirmada por biopsia, a quienes se administró Gardasil ${ }^{\circledR}$ en esquema estándar de 3 dosis y se midió la seroactividad de la proteína de cápside viral L1 de los serotipos 6 y 11 , con un seguimiento promedio de 4 años. Los resultados mostraron que la media de anticuerpos reactivos contra VPH aumentó en forma significativa de 1.125 MFI (Mean Fluorescense Intensity) previo a la vacuna, a $4.690 \mathrm{MFI}$. Este aumento de seroactividad ocurrió independiente de la edad, tiempo de inicio, severidad de la enfermedad y serotipo de VPH. Sin embargo, se concluye que si bien la vacuna tetravalente podría ser efectiva en el tratamiento de la PRR dada su cuantiosa respuesta inmunológica, es necesario un ensayo aleatorizado para evaluar el efecto de la vacuna en el curso clínico de la enfermedad.

Otro estudio de serie de casos realizado en Japón por Makiyama y colli en 2016, incluyó a 12 pacientes de sexo masculino entre 32 y 74 años con 
papilomatosis laríngea recurrente a quienes, luego de una resección del papiloma mediante láser, se les administró Gardasil ${ }^{\circledR}$ en esquema estándar de 3 dosis. Se midieron los títulos de los serotipos 6 , 11,16 y 18, antes y siete meses posteriores a la administración de la vacuna. Los resultados mostraron que hubo un aumento significativo de los títulos de anticuerpos posterior a la administración de la vacuna en hombres posadolescentes (desde menor a $11 \mathrm{mMu} / \mathrm{mL}$ en promedio a $597,3 \mathrm{mMu} /$ $\mathrm{mL}$ para el serotipo 6; desde menor a $8 \mathrm{mMu} / \mathrm{mL}$ a $687,8 \mathrm{mMu} / \mathrm{mL}$ para el serotipo 11; desde menor a $11 \mathrm{mMu} / \mathrm{mL}$ a $3.167,1 \mathrm{mMu} / \mathrm{mL}$ para el serotipo 16 ; y desde menor a $10 \mathrm{mMu} / \mathrm{mL}$ a $504,2 \mathrm{mMu} / \mathrm{mL}$ para el serotipo 18).

Finalmente, un estudio reciente prospectivo no randomizado realizado en Alemania por Goon y col ${ }^{19}$ publicado en 2017 incluyó a 12 pacientes con diagnóstico histopatológico de VPH y serotipo 6 y 11 a quienes se les ofreció tratamiento quirúrgico variable (láser, microdebridador, bisturí en frío) y se les administró Gardasil ${ }^{\circledR}$ según esquema de 3 dosis. Se registró el número de intervenciones quirúrgicas para el tratamiento de recurrencias antes y después de la aplicación de la vacuna. En sus resultados, los autores reportan una disminucion de 7,07 veces en las tasas de incidencia de papilomatosis que requieren intervención quirúrgica desde el período previo a la vacunación $(47,44 / 1.000$ pacientes-meses) en comparación con el el período posterior a la vacunación (6,71/1.000 pacientes-meses).

\section{DISCUSIÓN}

Si bien los resultados antes expuestos parecieran favorecer el uso de la vacuna tetravalente para el tratamiento de la papilomatosis respiratoria recurrente, consideramos relevante tener en cuenta otros aspectos para la toma de decisiones. En primer lugar, ninguno de los estudios previamente analizados corresponde a un ensayo aleatorizado, lo que reduce la certeza de la evidencia obtenida en forma considerable. Además, los estudios previamente analizados realizan más de una intervención simultánea (como el uso de cidofovir y/o cirugía), lo que dificulta el análisis de causalidad en los desenlaces obtenidos. Por otro lado, el número de pacientes de los estudios es bajo, lo que dificulta un análisis significativo de los resultados obtenidos.

Como se expuso previamente, la vacuna actuaría en pacientes ya infectados aumentando la respuesta inmune contra el virus y previniendo la reinfección. Consideramos que el seguimiento por 1 año no es suficiente para evaluar la recurrencia, por lo que se requieren estudios con seguimiento a más largo plazo. Existe una alta probabilidad de que futuras investigaciones cambien las conclusiones de esta revisión, dada la alta controversia respecto al tema.

En Chile la vacuna tetravalente Gardasil ${ }^{\circledR}$ se encuentra incluida en el Plan Nacional de Inmunizaciones (cubierta por el Estado), desde 2014 para niñas de 9 años ${ }^{20}$ con dos dosis separadas por 1 año de diferencia, con el objetivo de protegerlas contra el cáncer cervicouterino y condilomas acuminados, previo a la exposición sexual. El esquema de dos dosis adoptado en nuestro país se justifica por la utilización de vacuna Cervarix ${ }^{\circledR}$, cuyo esquema de dos dosis ha sido aprobado por la Agencia de Medicamentos Europea (EMA) para niñas de 9 a 14 años ${ }^{21}$, basado en dos estudios de no inferioridad que la comparan con el esquema de tres dosis (HPV 40 y HPV 70)22,23. La población no incluida en el Plan Nacional de Inmunizaciones, sin embargo, debe adquirirla de forma particular, teniendo un costo considerable en el mercado de alrededor de $\$ 70.000$ (CLP) por dosis. Existe además una vacuna nonavalente, Gardasil $9^{\circledR}$ que agrega cobertura contra los serotipos $31,33,45,52$ y 58 , aprobada por la FDA desde 2014, con estudios de no inferioridad a la tetravalente y efectividad en generar inmunogenicidad contra los serotipos no incluidos en las coberturas anteriores ${ }^{24}$ que aún no ha sido estudiada para PRR.

Dada la información obtenida en la literatura, el uso de la vacuna sería efectiva en el tratamiento de la PRR al lograr aumentar en forma significativa los títulos de anticuerpos anti-VPH y disminuir la recurrencia de la enfermedad, con pocos efectos adversos significativos, lo cual inclinaría el balance riesgo-beneficio a favor de la intervención. 


\section{CONCLUSIÓN}

La evidencia actualmente disponible, proveniente de estudios primarios de baja calidad en cuanto a su diseño, apunta a que la vacuna tetravalente es posiblemente efectiva como tratamiento en pacientes con papilomatosis respiratoria recurrente logrando disminuir el número de recurrencias, aumentar

\section{BIBLIOGRAFÍA}

1. Forman D, de Martel C, Lacey CJ, Soerjomataram I, Lortet-Tieulent J, Bruni L, Vignat J, Ferlay J, Bray F, Plummer M, et al. Global bur- den of human papillomavirus and related diseases. Vaccine 2012; 30: F12-23.

2. Bonagura VR, Hatam LJ, Rosenthal DW, DeVoti JA, Lam F, Steinberg BM, Abramson AL. Recurrent respiratory papillomatosis: a complex defect in immune responsiveness to human papillomavirus-6 and -11. APMIS. 2010; 118: 455-70.

3. Abramson AL, Steinberg BM, Winkler B. Laryngeal papillomatosis: clinical, histopathologic and molecular studies. Laryngoscope 1987; 97: 678-85.

4. DeRKAY CS. Task force on recurrent respiratory papillomas. A preliminary report. Arch Otolaryngol Head Neck Surg 1995; 121: 1386-91.

5. Armstrong LR, Derkay CS, Reeves WC. Initial results from the national registry for juvenileonset recurrent respiratory papillomatosis. RRP Task Force. Arch Otolaryngol Head Neck Surg 1999; 125: 743-8.

6. Mammas I, Sourvinos G, Spandidos D. The 'Trojan horse' oncogenic strategy of HPVs in childhood. Future Virol 2013; 8: 801-8.

7. Wiatrak B, Wiatrak D, Broker T, Lewis L. Recurrent Respiratory Papillomatosis: A Longitudinal Comparing Severity With Human Papilloma Viral Types 6 and 11 and Other Risk Factors in a Large Pediatric Population. Laryngoscope 2004; 114 : 1-23.

8. Zhal L, Tumban E. Gardasil-9: A global survey of projected efficacy. Antiviral Res 2016; 130: 101-9.

9. Doyle D, Gianoli G, Espinola T, Miller R. Recurrent Respiratory Papillomatosis: juvenile versus adult el intervalo libre de cirugías, obtener remisión completa 0 parcial de la enfermedad y aumentar significativamente los títulos de anticuerpos anti-VPH. Estos efectos serían más considerables en pacientes de sexo masculino. No obstante, aún es necesario mayor estudio con ensayos clínicos randomizados con seguimiento a mayor plazo para una mejor evidencia respecto a los resultados observados.

forms. Laryngoscope 1994; 104: 523-7.

10. José Veríssimo Fernandes and Thales Allyrio Araújo de Medeiros Fernandes. Human Papillomavirus: Biology and Pathogenesis, Human Papillomavirus and Related Diseases - From Bench to Bedside A Clinical Perspective, Dr. Davy Vanden Broeck (Ed.), InTech. 2012.

11. Pomfret TC, Gagnon JM Jr, Gilchrist AT. Quadrivalent human papilloma- virus (HPV) vaccine: a review of safety, efficacy, and pharmacoeconomics. J Clin Pharm Ther 2011; 36: 1-9.

12. Giuliano AR, Lazcano-Ponce E, Villa L, et al. Impact of baseline covariates on the immunogenicity of a quadrivalent (types $6,11,16$, and 18) human papillomavirus virus-like-particle vaccine. $J$ Infect Dis 2007; 196: 1153-62.

13. Tuon Pian Gi RE, San Giorgi MR, Pawlita M, Michel A, van Hemel bM, Schuuring EM, van den Heuvel eR, van der Laan BF, Dikkers FG. Immunological response to quadrivalent HPV vaccine in treatment of recurrent respiratory papillomatosis. Eur Arch Otorhinolaryngol2016; 273: 3231-6.

14. Luo W, Zhang S, Zhou Y, Wang C, Yang L, Qiu J. Safety and Immunogenicity of Quadrivalent HPV Vaccine: A Meta-analysis. Chinese Journal of Evidence-Based Medicine 2015; 1: 47-53.

15. Slade BA, Leidel L, Vellozzi C, et al. Postlicensure safety surveillance for quadrivalent human papillomavirus recombinant vaccine. J Am Med Assoc 2009; 302: 750-7.

16. Chirila M, Bolboaca SD. Clinical efficiency of quadrivalent HPV (types 6/11/16/18) vaccine in patients with recurrent respiratory papillomatosis. Eur Arch Otorhinolaryngol2014; 271: $1135-42$.

17. Young DL, Moore MM, Halstead LA. The use of the quadrivalent humanpapillomavirus vaccine 
(gardasil) as adjuvant therapy in the treatment of recurrent respiratory papilloma. J Voice 2015; 29: 223-9.

18. Makiyama K, Hirai R, Matsuzakı H. Gardasil Vaccination for Recurrent Laryngeal Papillomatosis in Adult Men: First Report: Changes in HPV Antibody Titer. J Voice 2017; 31: 104-6.

19. Goon PK, Scholtz L, Sudhoff H. Recurrent Respiratory Papillomatosis (RRP)-Time for a Reckoning? Laryngoscope Investigative Otolaryngology 2017; 2: 184-6.

20. Calendario de vacunación 2017. PNI. Ministerio de Salud. Gobierno de Chile. Disponible en: http://vacunas.minsal.cl/informacion-a-lacomunidad/calendario-de-vacunacion.

21. EMA. European Medicines Agency, Committee for Medicinal Products for Human Use (CHMP) Assessment report EMA/789820/2013 Cervarix 2013.
22. GlaxoSmithKline. Evaluation of the safety and immunogenicity of GlaxoSmithKline Biologicals' HPV vaccine 580299 when administered in healthy females aged $9-25$ years using an alternative schedule and an alternative dosing as compared to the standard schedule and dosing. 2013 Disponible en: http://download.gskclinicalstudyregister.com/ files/ebe3f40a-ef27-469c-8874-35053b5a80d7.

23. GlaxoSmithKline. Immunogenicity and safety study of GlaxoSmithKline (GSK) Biologicals' human papillomavirus (HPV)-16/18 L1 AS04 vaccine when administered according to alternative 2-dose schedules in 9 - 14 year old females. 2013 Disponible en: http://download.gsk-clinicalstudyregister.com/ files/1ae03c85-a5fe-4339-a1e1- 03a3d97f6793.

24. Herrero R, Gonzalez P, Markowitz. Present status of human papillomavirus vaccine development and implementation. Lancet Oncol 2015; 16: e206-16. 